\title{
Conductance quantization in multiwalled carbon nanotubes
}

\author{
Ph. Poncharal ${ }^{1}$, St. Frank ${ }^{1}$, Z.L. Wang ${ }^{2}$, and W.A. de Heer ${ }^{1}$ \\ ${ }^{1}$ School of Physics, Georgia Institute of Technology, Atlanta, GA 30332, USA \\ ${ }^{2}$ School of Materials Science and Engineering, Georgia Institute of Technology, Atlanta, GA 30332, USA
}

Received: 2 September 1998 / Received in final form: 8 February 1999

\begin{abstract}
We present results of carbon nanotube conductance measurements. The experiments were performed using an scanning probe microscope (SPM) system where a carbon nanotube fiber is connected to the SPM tip and then lowered into a liquid mercury contact. Experiments were also performed using a modified transmission electron microscope (TEM) specimen holder supplied with piezo and micrometer positioning system. Thus the contacting process of the nanotubes with the mercury could be monitored while simultaneously recording the conductance. These measurements and observations confirm previously reported conductance quantization (Frank et al.: Science 280, 1744 (1998)) of the nanotubes while providing additional details concerning the mercury nanotube contacts. We also report conductance versus voltage characteristics of carbon nanotubes.
\end{abstract}

PACS. 85.40.-e Microelectronics: LSI, VLSI, ULSI: integrated circuit fabrication technology - 61.46.+w Clusters, nanoparticles, and nanocrystalline materials

\section{Introduction}

Transport in multiwalled carbon nantotubes has been investigated using from the simplest to the most complex of experimental approaches [1]. In the simplest cases, the conductance of the nanotube is deduced from measurements of bulk samples or films made of nanotubes [2]. In the more complex cases, the contacts are lithographically patterned on a substrate and a single nanotube is then connected to them. In this manner, two, three and four probe measurements of carbon nanotubes have been made [3-5]. The electrical conductivity has also been measured using scanning probe methods, where a nanotube is anchored at one end to a conducting lead and the conducting tip of a scanning force microscope is slid along the tube facilitating conductance versus length measurements [6].

The measurements gave very diverse results with resistances and resistivities spanning several orders of magnitude [3-5]. In fact in one case it appeared as if the current was flowing in a tortuous manner through the tube in a four probe measurement, since the voltage measured at the inner conductors was in the opposite direction compared to the voltage applied to the outer conductors [3].

In contrast, transport theory predicts entirely different behavior: the conductance of single wall nanotubes should be quantized $[7,8]$ and either 0 or $2 G_{0}$, where $G_{0}=2 \mathrm{e}^{2} / h=1 /(12.9 \mathrm{k} \Omega)[9-12]$. This logically should be a lower conductance bound for multiwalled nanotubes. In fact the measured conductances are usually much smaller than $G_{0}$ (i.e. typically on the order of $1 /(100 \mathrm{k} \Omega) \approx$ $\left.G_{0} / 10\right)$. Hence the contacts between the nanotubes and the leads were suspect. In principle, four probe measurements should be immune to contact resistances, however in these cases it may be that the gold/nanotube contacts are not well reproducible and hence compensation is imperfect.

Another problem lies with the processing of the carbon nanotubes. In principle, the nanotubes are quite robust, due the extreme durability of the graphitic bond. However, ultrasonification and other processes typically used to separate and manipulate the nanotubes can be destructive, which may affect their transport properties $[13,14]$.

\section{Experiment}

A better method to contact the nanotubes in pristine condition is therefore to be sought. To this end we used the nanotube fibers which were recovered from the nanotube deposits of arc produced carbon nanotubes [15]. These fibers consist mainly of densely packed multiwalled nanotubes and are about $1 \mathrm{~mm}$ long and $0.1 \mathrm{~mm}$ wide. The fibers are attached to a gold wire (using silver colloid) and attached to the piezo of a SPM (Park Instruments Autoprobe). The assembly is lowered towards a liquid mercury filled copper cup so that the conductance can be measured. As we have previously demonstrated [1] the conductance abruptly jumps from 0 to values close to $1 G_{0}$. This value might be sligtly less in some cases (up to $14 \mathrm{k} \Omega$ ), which is what expected if the nanotube is not in perfect contact with the fiber. When the fiber is submerged even further the conductance increased stepwise (typically in units of $1 G_{0}$, Fig. 1), until ultimately contact was made with the stock, where the residual conductance is about $1000 G_{0}$, 


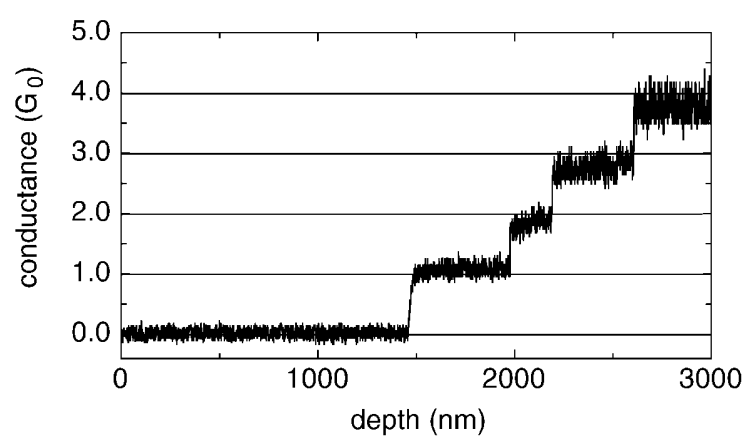

Fig. 1. Conductance of a carbon nanotube fiber bundle which is pushed into a liquid mercury surface (after completion of the cleaning procedure) using the SPM method. The applied voltage is $100 \mathrm{mV}$. According to our survey of the protruding nanotubes, the typical diameters are in the range of 15 to $35 \mathrm{~nm}$. The four succesive steps are due to four nanotubes which protrude from the fiber stalk. The nanotubes have different lengths and hence they make contact with the mercury at different times during the approach. Each nanotube contributes $1 G_{0}$ to the conductance and hence the steps. When the fiber is pushed even deeper into the mercury, so that the stalk of the fiber makes contact with the mercury, the conductance is on the order of $1000 G_{0}$.

(on the order of 10-20 $\Omega$ ). The measurements are highly reproducible for each sample where the fiber can be lowered into the mercury and retracted for many cycles where the pattern of steps repeats.

However this reproducibility only occurs after several thousand initial cycles when using a freshly constructed nanotube contact. Initial residual conductances are much smaller (as low as $0.02 G_{0}$ ), and poorly quantized steps which do not reproduce often appear. Moreover, the initial curing cycles leave a visible black deposit on the mercury surface which is evidently composed of fragments from the fiber. Next, the contact position with the mercury is displaced to a clean area, and the experiment is repeated after which invariably quantized conductance steps are observed (note that often presteps of $0.5 G_{0}$ are observed, see Frank [1]). From these observations we concluded that the curing procedure had a cleansing effect of the tip of the bundle whereby poorly connected tubes were separated from the tip leaving only well anchored tubes.

More recent experiments were performed in situ, in the electron microscope, using a specially designed specimen holder as shown in Fig. 2. With this device, a nanotube fiber can be moved towards a mercury droplet which is suspended at the end of a copper wire. The fiber can be brought into contact with the mercury droplet either by micrometer control or with using the piezo actuator. In these experiments we were able to repeatedly make and break contact between the tip of the fiber and the mercury, so that the carbon nanotube which made the contact could be identified.

This in situ configuration also allowed us to monitor the cleaning process described above: the nanotubes on a fresh tip are covered with small graphitic particles and loose agglomerations of nanotubes, as well as poorly connected

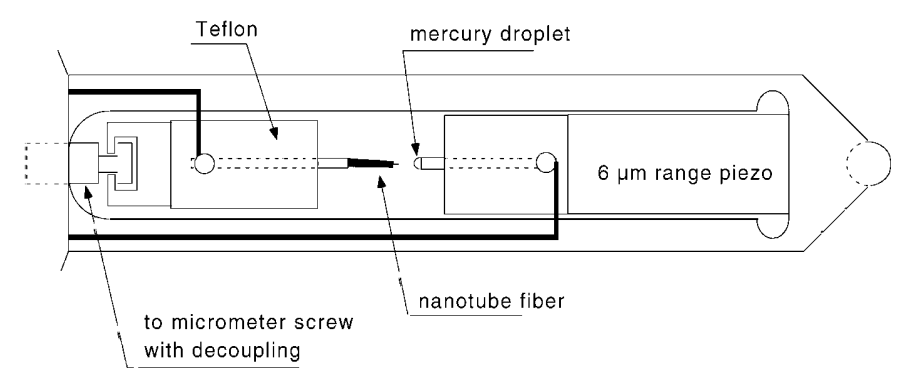

Fig. 2. TEM sample holder for in situ nanotube experiments (length is $3.5 \mathrm{~cm}$, width $0.7 \mathrm{~cm}$ ). The nanotube fiber which is attached to a gold wire (left) can be manually moved toward the mercury droplet (right) by means of a micrometer. The piezo actuator is used for the final approach. The micrometer shaft can be decoupled from the sample to inhibit vibrations.

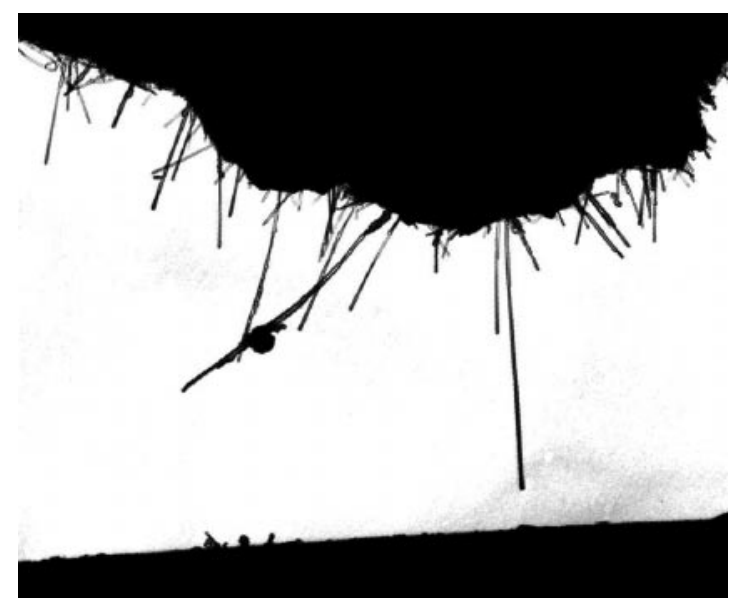

Fig. 3. Transmission electron micrograph of a nanotube approaching the mercury surface using the sample holder described in Fig. 2. The long vertical nanotube is $2.2 \mu \mathrm{m}$ long and $25 \mathrm{~nm}$ in diameter. A graphitic particle is attached to one of the nanotubes. Small graphitic particles which have detached from the fiber adhere to the mercury surface.

nanotubes, which are pushed away during submerging cycles. What remains is a compact stalk from which straight nanotubes protrude. At this time the mercury surface is covered with patches of nanotubes and small graphitic particles, which have been separated from the nanotube fiber during the dipping process. In order to continue measurements on clean mercury requires removing the sample from the microscope and replacing the mercury contact. We used several pre-cleaned fibers which were clearly distinguished from the untreated ones in that the nanotubes tend to stick out perpendicular to the surface, and only very few graphitic particles are observed on the nanotubes (Fig. 3).

A difficulty important to overcome was to have the parallax adjustable so that the contact point could be observed (i.e. so that this point was not obscured from the electron beam). Another difficulty relates to charge accumulation on the sample holder which caused the images to be unstable and caused blurring in many of the photographs. 


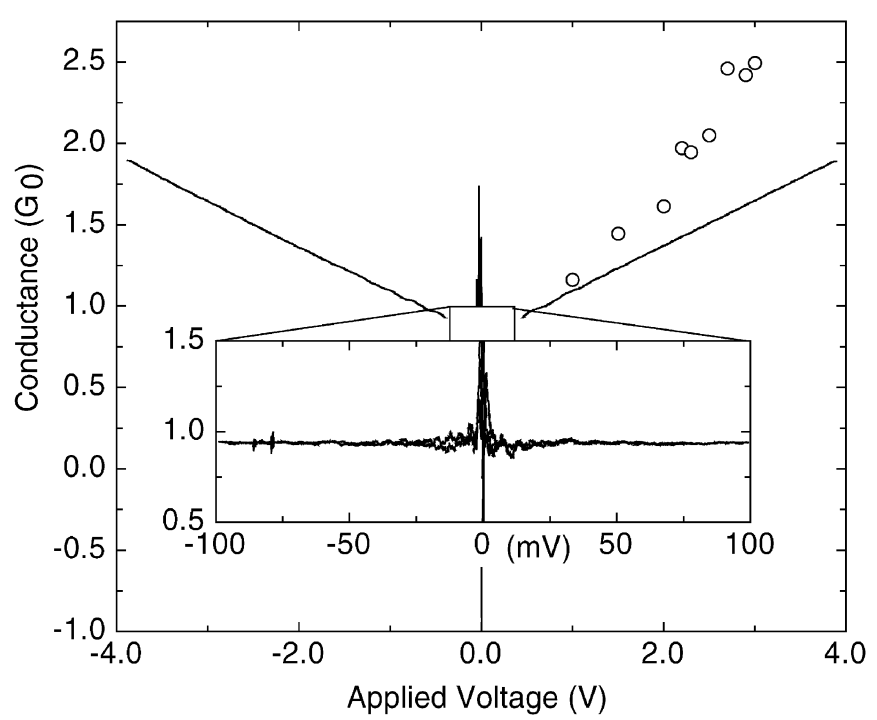

Fig. 4. Typical nanotube conductance versus voltage characteristics. The nanotube conductance is constant and close to integer values for low applied voltages (typically up to $100 \mathrm{mV}$, see inset). At higher voltages, the conductance increases linearly. The solid curve, recorded using the SPM in air, exhibits an increase of $0.25 G_{0} /$ Volt. The circles have been recorded in the TEM and also show a linear increase $\left(0.5 G_{0} /\right.$ Volt $)$. The slopes are sample dependent and range from between 0.25 to $1 G_{0}$ /Volt. In the TEM measurement, contact was broken at $3 \mathrm{~V}$ (corresponding to a current of $0.6 \mathrm{~mA}$ ) probably due to the Lorentz force. The SPM measured nanotube was stable at $4 \mathrm{~V}$ $(0.5 \mathrm{~mA})$. In the TEM we have observed nanotubes $1 \mu \mathrm{m}$ in length which were stable with currents on the order of $0.5 \mathrm{~mA}$ passing through them.

These TEM experiments allowed us to directly verify that the initial conductance was indeed made by single nanotubes protruding out of the fiber. Once in contact, we observed that by applying a sufficiently high voltage (usually about $3-5 \mathrm{~V}$ ), one tube would suddenly jump away and the conductance would drop to 0 . The currents are then typically $0.5 \mathrm{~mA}$ which gives extraordinarily large current densities (the observed nanotubes that survive the cleaning process have diameters between 15 to $30 \mathrm{~nm}$ ). In fact, even with this high current density, the nanotube may not have burnt, since there is evidence that it was simply pushed away due to the large Lorentz force causing the contact to be interrupted (note that the objective lens of the microscope supplies a field of about $1 \mathrm{~T}$ perpendicular to the nanotube).

We also observed that not all nanotubes conduct. In many cases nanotubes clearly contacted the mercury yet the resistance was very large $(>10 \mathrm{M} \Omega)$. This may reflect the theoretical prediction that only one in three tubes conduct due to the helicity effect on the conductivity in single wall nanotubes.

Quantum conductance is expected to be independent of the nanotube diameter but should scale with the number of layers. In order to explain the observed effects, we assume that only the outer layer of the nanotube participates in the electrical transport [1].
Conductance versus applied voltage measurements were performed both in air and in the microscope. Typically a $\mathrm{V}$ shaped flat bottomed curve is observed (see Fig. 4), where a flat bottom occurs between $-0.1 \mathrm{~V}$, $+0.1 \mathrm{~V}$. We do not have an adequate explanation for this effect. However it may be related to the tunneling of electrons from one layer to the next. It should be noted that the loss of quantization at these very large voltages is not in contradiction to the theory, since quantization is only expected for low bias voltages.

\section{Conclusion}

The new results presented here demonstrate the feasibility of performing experiments where nanotubes are manipulted with direct visual control under the TEM. The experiments confirm previous conclusions: the conductances of individual nanotubes can be measured in a fibermercury contact experiment. The conductances measured under TEM are consistant with the $1 G_{0}$ per tube reported in [1].

The conductance versus voltage measurements demonstrate that the conductance is constant for low bias voltages $(-0.1$ to $+0.1 \mathrm{~V})$ and increases linearly for larger voltages with a slope which is sample dependent. We are not aware of an explanation of this effect.

This work was supported by the ARO under project DAAG5597-1-0133. S.F. thanks the Alexander von Humbolt Foundation for financial support. The authors thank U. Landman, R.L. Whetten and P. First for helpful discussions.

\section{References}

1. S. Frank, P. Poncharal, Z.L. Wang, W.A. de Heer: Science 280, 1744 (1998)

2. G. Baumgartner et al.: Phys. Rev. B 55, 6704 (1997)

3. T.W. Ebbesen et al.: Nature 382, 54 (1996)

4. L. Langer et al.: Phys. Rev. Lett. 76, 479 (1996)

5. A. Bachtold, C. Strunk, C. Schonenberger, J.-P. Salvetat, L. Forro: Electrical properties of single carbon nanotubes, ed. by H. Kuzmany, J. Fink, M. Mehring, S. Roth, International Winterschool on Electronic Properties of novel Materials "Molecular Nanostructures" (AIP, New York 1998) p. 65

6. H. Dai, E.W. Wong, C.M. Lieber: Science 272, 523 (1996)

7. R. Landauer: Philos. Mag. 21, 863 (1970)

8. S. Datta: Electronic transport properties in mesoscopic systems (Cambridge University Press, Cambridge 1995)

9. J.W. Mintmire, B.I. Dunlap, C.T. White: Phys. Rev. Lett. 68, 631 (1992)

10. J.W. Mintmire, C.T. White: Carbon 33, 893 (1995)

11. W. Tian, S. Datta: Phys. Rev. B 49, 5097 (1994)

12. L. Chico, L.X. Benedict, S.G. Louie, M.L. Cohen: Phys. Rev. B 54, 2600 (1996)

13. K.B. Shelimov et al.: Chem. Phys. Lett. 282, 429 (1998)

14. D. Ugarte: Z. Phys. D 26, 150 (1993)

15. T.W. Ebbesen, P.M. Ajayan: Nature 358, 220 (1992) 